\title{
Asymptotic $C^{*}$-Algebras from $\mathbb{Z}^{k}$-Actions on Higher Rank Graphs
}

\author{
Inhyeop Yi \\ Department of Mathematics Education, Ewha Womans University, Seoul 120-750, Republic of Korea \\ Correspondence should be addressed to Inhyeop Yi; yih@ewha.ac.kr
}

Received 17 March 2013; Accepted 5 June 2013

Academic Editor: Baodong Zheng

Copyright ( 2013 Inhyeop Yi. This is an open access article distributed under the Creative Commons Attribution License, which permits unrestricted use, distribution, and reproduction in any medium, provided the original work is properly cited.

For a dynamical system arising from $\mathbb{Z}^{k}$-action on a higher rank graph with finite vertex set, we show that the semidirect product of the asymptotic equivalence relation groupoid is essentially principal if and only if the $k$-graph satisfies the aperiodic condition. Then we show that the corresponding asymptotic Ruelle algebra is simple if the graph is primitive with the aperiodic condition.

\section{Introduction}

In [1], Kumjian and Pask constructed $\mathbb{Z}^{k}$-action dynamical systems on higher rank graphs, which are higher dimensional analog of subshifts of finite type in symbolic dynamics. Naturally their dynamical systems exhibit many of the same dynamical properties in not only subshifts of finite type but also Smale spaces studied by Putnam [2], Putnam and Spielberg [3], and Ruelle [4,5]. Putnam associated several groupoid $C^{*}$-algebras to Smale spaces using asymptotic, stable, and unstable equivalence relations generated by hyperbolic structures of Smale spaces [2]. Ruelle algebras from stable and unstable equivalence relations may be considered as higher dimensional analogs of Cuntz-Krieger algebras [2, $3,6]$. As in the case of irreducible Smale spaces, Kumjian and Pask built $C^{*}$-algebras from the stable and unstable equivalence relations on their dynamical systems and the related Ruelle algebras [1]. Then they showed that stable Ruelle algebra $R_{s}$ of a higher rank graph is strongly Morita equivalent to the graph $C^{*}$-algebra and that $R_{s}$ is simple, nuclear, and purely infinite if the graph satisfies the aperiodic condition. Although Kumjian and Pask showed that the groupoid algebra from asymptotic equivalence relation is a simple $A F$ algebra if the graph is primitive, they left Ruelle algebra $R_{a}$ from asymptotic equivalence relation behind, and it remains unclear whether or not $R_{a}$ is simple. Here, we show that $R_{a}$ is simple if the corresponding higher rank graph is primitive with aperiodic condition (Theorem 16). For this purpose, we first review the definitions and necessary properties of $k$-graphs and $\mathbb{Z}^{k}$-action in Section 2. Then we follow Hou and Chen [7] to study structures of groupoids from asymptotic equivalence relation, and we obtain sufficient conditions for $R_{a}$ to be simple in Section 3 .

\section{Dynamical Systems on Higher Rank Graphs}

In this section, we briefly review the definitions and notations for $k$-graphs and $\mathbb{Z}^{k}$-action on those established by Kumjian and Pask in [1].

Definition 1 (see $[1,8])$. A $k$-graph is a pair $(\Lambda, d)$, where

$$
\Lambda=(\operatorname{Obj}(\Lambda), \operatorname{Hom}(\Lambda), r, s),
$$

is a countable small category and $d: \Lambda \rightarrow \mathbb{N}^{k}$ is a morphism, called the degree map, satisfying the factorization property. For every $\lambda \in \Lambda$ and $m, n \in \mathbb{N}^{k}$ with $d(\lambda)=m+n$, there exist unique elements $\mu, v \in \Lambda$ such that

$$
d(\mu)=m, d(\nu)=n, \quad \lambda=\mu \nu .
$$

Every $\lambda \in \Lambda$ is called a path. For every nonzero $n \in \mathbb{N}^{k}$, by the factorization property we may define $\Lambda^{n}=d^{-1}(n)$ and identify $\Lambda^{0}$ with $\operatorname{Obj}(\Lambda)$. Let $r, s: \Lambda \rightarrow \Lambda^{0}$ be the range and source maps. We abbreviate $(\Lambda, d)$ to $\Lambda$ when there is no confusion. 
Definition 2 (see $[1,8]$ ). A $k$-graph $\Lambda$ is called primitive if there is a nonzero $p \in \mathbb{N}^{k}$ so that for every $u, v \in \Lambda^{0}$ there is a $\lambda \in \Lambda^{p}$ such that $u=r(\lambda)$ and $v=s(\lambda)$.

Standing Assumption (see [1]). For every $n \in \mathbb{N}^{k}$, the restrictions of $r$ and $s$ on $\Lambda^{n}$ are surjective and finite to one.

2.1. $\mathbb{Z}^{k}$-Actions on $k$-Graphs $($ See $[1,2])$. Suppose that $(\Delta, d)$ is a $k$-graph defined by

$$
\Delta=\left\{(m, n) \mid m, n \in \mathbb{Z}^{k}, m \leq n\right\},
$$

with the structure maps

$$
\begin{gathered}
(l, m) \cdot(m, n)=(l, n), \quad r(m, n)=m, \\
s(m, n)=n, \quad d(m, n)=m-n .
\end{gathered}
$$

Let $\Lambda$ be a $k$-graph, and let the corresponding two-sided infinite path space be the set

$$
\Lambda^{\Delta}=\{x: \Delta \longrightarrow \Lambda \mid x \text { is a } k \text {-graph homomorphism }\} .
$$

Then, $\Lambda^{\Delta}$ is a zero-dimensional space consisting of "twosided" paths on $\Lambda$.

A topology is endowed on $\Lambda^{\Delta}$ where its basis is given by

$$
Z(\lambda, n)=\left\{x \in \Lambda^{\Delta} x \mid(n, n+d(\lambda))=\lambda\right\}
$$

with $n \in \mathbb{Z}^{k}$ and $\lambda \in \Lambda$. It is not difficult to check that $\Lambda^{\Delta}$ is compact (locally compact, resp.) if $\Lambda^{0}$ is finite (infinite, resp.) so that $\Lambda^{\Delta}$ is a metrizable space. A metric $\rho$ on $\Lambda^{\Delta}$ is defined as follows: for $e=(1, \ldots, 1) \in \mathbb{Z}^{k}$ and $j \in \mathbb{N}$, let $\theta_{j} \in \Delta$ be the element $(-j e, j e)$. Given $x, y \in \Lambda^{\Delta}$, set

$$
h(x, y)= \begin{cases}1 & x(0) \neq y(0) \\ 1+\sup \left\{j \mid x\left(\theta_{j}\right)=y\left(\theta_{j}\right)\right\} & \text { otherwise }\end{cases}
$$

Then, for a fixed number $r \in(0,1)$, a metric $\rho$ is defined by the formula

$$
\rho(x, y)=r^{h(x, y)} \quad \text { for } x, y \in \Lambda^{\Delta} .
$$

The metric topology is the same as the one generated by cylinder sets. And we remark that the cylinder set $Z(\lambda, n)$ is always nonempty because of our standing assumption.

Let $\sigma$ be the action of $\mathbb{Z}^{k}$ on $\Lambda^{\Delta}$ by the homeomorphism $\sigma^{p}: \Lambda^{\Delta} \rightarrow \Lambda^{\Delta}, p \in \mathbb{Z}^{k}$, defined by

$$
\left(\sigma^{p} x\right)(m, n)=x(m+p, n+p) .
$$

Theorem 3 (see [1]). The $\mathbb{Z}^{k}$-action $n \mapsto \sigma^{n}$ on $\Lambda^{\Delta}$ is expansive in the sense that there is an $\epsilon>0$ such that, for $x, y \in \Lambda^{\Delta}, \rho\left(\sigma^{n}(x), \sigma^{n}(y)\right)<\epsilon$ for every $n \in \mathbb{Z}^{k}$ implies $x=y$. Moreover, if $\Lambda$ is primitive, then $\sigma$ is topologically mixing.
2.2. Groupoids on $\left(\Lambda^{\Delta}, \sigma, \rho\right)$. We review Kumjian and Pask's construction of the asymptotic groupoid $G_{a}$ and its corresponding $C^{*}$-algebra $C^{*}\left(G_{a}\right)$ and Ruelle algebra $R_{a}$ on $\left(\Lambda^{\Delta}, \sigma, \rho\right)$, where $\rho$ is the metric defined on $\Lambda^{\Delta}$ as in (8).

For a second countable locally compact groupoid $G$, we denote the source map and the range map of $G$ by $s$ and $r$, respectively, and $G^{0}$ is the unit space of $G$. We refer to [9] for more detailed results on groupoids and groupoid $C^{*}$ algebras.

As in the case of subshifts of finite types, the asymptotic, stable, and unstable equivalence relations on $\Lambda^{\Delta}$ are defined as follows [1]: given $x, y \in \Lambda^{\Delta}$, we define

$$
\begin{aligned}
& x \sim_{a} y \quad \text { if } \lim _{|j| \rightarrow \infty} \rho\left(\sigma^{j e}(x), \sigma^{j e}(y)\right)=0, \\
& x \sim_{s} y \quad \text { if } \lim _{j \rightarrow \infty} \rho\left(\sigma^{j e}(x), \sigma^{j e}(y)\right)=0, \\
& x \sim_{u} y \quad \text { if } \lim _{j \rightarrow-\infty} \rho\left(\sigma^{j e}(x), \sigma^{j e}(y)\right)=0 .
\end{aligned}
$$

Then, we denote the asymptotic, stable, and unstable equivalence classes of $x$ by $V^{a}(x), V^{s}(x)$, and $V^{u}(x)$, respectively.

Remark 4 (see [1, Remark 3.6]). For $x, y \in \Lambda^{\Delta}, x \sim_{a} y$ if and only if there is an $m \in \mathbb{N}^{k}$ such that for every $n \geq m$ we have

$$
x(m, n)=y(m, n), \quad x(-n,-m)=y(-n,-m) .
$$

The asymptotic equivalence relation gives rise to a groupoid

$$
G_{a}=\left\{(x, y) \in \Lambda^{\Delta} \times \Lambda^{\Delta}: x \sim{ }_{a} y\right\}
$$

whose unit space is identified with $\Lambda^{\Delta}$, and structure maps are the natural ones. To give a topology on $G_{a}$, we construct conjugate homeomorphisms as in Ruelle [5]: for $(x, y) \in G_{a}$, let $m \in \mathbb{N}^{k}$ be as in Remark 4 and define finite paths $\lambda=$ $x(-m, m)$ and $\nu=y(-m, m)$. We recall that the cylinder sets

$$
\begin{aligned}
& Z(\lambda,-m)=\left\{z \in \Lambda^{\Delta}: z(-m, m)=x(-m, m)\right\}, \\
& Z(\nu,-m)=\left\{w \in \Lambda^{\Delta}: w(-m, m)=y(-m, m)\right\},
\end{aligned}
$$

are compact open subsets of $\Lambda^{\Delta}$. Since $x$ and $y$ satisfy the equalities in Remark 4, when we define $\varphi: Z(\lambda,-m) \rightarrow$ $Z(\nu,-m)$ by

$$
\begin{gathered}
\varphi(x)=y, \varphi(z)(m, n)=z(m, n), \\
\varphi(z)(-n,-m)=z(-n,-m),
\end{gathered}
$$

for all $z \in Z(\lambda,-m)$ and $n \geq m, \varphi$ is a bijective map by the factorization property and $z \sim_{a} \varphi(z)$ by the definition of $\varphi$ and Remark 4. It is easy to check that $\varphi$ is continuous so that it is a homeomorphism.

A basis for the topology of $G_{a}$ is given by the compact open sets

$$
\left\{(\varphi(z), z): z \in Z(\lambda,-m), m \in \mathbb{N}^{k}\right\}
$$


Then, $G_{a}$ is a second countable, locally compact, Hausdorff, $r$ discrete, and principal groupoid under this topology, and the counting measure is a Haar system $[2,7,8]$. The path space $\Lambda^{\Delta}$ is identified with the unit space $G_{a}^{0}$ by the embedding map $x \mapsto(x, x)$.

To make the asymptotic Ruelle algebra, we need another groupoid: we imitate [2] to form the semidirect product $G_{a} \rtimes$ $\mathbb{Z}^{k}$ given by

$$
G_{a} \rtimes \mathbb{Z}^{k}=\left\{(u, n, v): u, v \in \Lambda^{\Delta}, n \in \mathbb{Z}^{k},\left(\sigma^{n}(u), v\right) \in G_{a}\right\},
$$

with the structure maps

$$
\begin{gathered}
s(u, n, v)=u, r(u, n, v)=v, \\
(u, n, v)(w, m, z)=(u, n+m, z) \quad \text { if } v=w, \\
(u, n, v)^{-1}=(v,-n, u) .
\end{gathered}
$$

It is not difficult to check that the map defined by $((x, y), n) \mapsto\left(x, n, \sigma^{n}(y)\right)$ gives a groupoid isomorphism between $G_{a} \times \mathbb{Z}^{k}$ and $G_{a} \rtimes \mathbb{Z}^{k}$. We transfer the product topology on $G_{a} \times \mathbb{Z}^{k}$ via this isomorphism so that $G_{a} \rtimes \mathbb{Z}^{k}$ is a locally compact, Hausdorff, and $r$-discrete groupoid, and the counting measure is a Haar system. The unit space is identified with $\Lambda^{\Delta}$ via the map $x \mapsto(x, 0, x)$.

Definition 5 (see $[1,2])$. Suppose that $\Lambda$ is a $k$-graph and that $G_{a}$ and $G_{a} \rtimes \mathbb{Z}^{k}$ are groupoids on $\Lambda$ defined previously. The asymptotic $C^{*}$-algebra of $\Lambda$ is the reduced groupoid $C^{*}$ algebra of $G_{a}$, denoted by $A(\Lambda)$. And the asymptotic Ruelle algebra of $\Lambda$ is the reduced groupoid $C^{*}$-algebra of $G_{a} \rtimes \mathbb{Z}^{k}$, denoted by $R_{a}(\Lambda)$.

\section{Main Results}

In this section, we first show that for a primitive $k$-graph, the groupoids $G_{a}$ and $G_{a} \rtimes \mathbb{Z}^{k}$ are minimal. Then, we show that if a $k$-graph $\Lambda$ is such that $\Lambda^{0}$ is finite, then the aperiodic condition is equivalent to essential principality of $G_{a} \rtimes \mathbb{Z}^{k}$. So the asymptotic $C^{*}$-algebra $A(\Lambda)$ is simple if $\Lambda$ is primitive, and the asymptotic Ruelle algebra $R_{a}(\Lambda)$ is simple if in addition $\Lambda$ satisfies the aperiodic condition.

Lemma 6. Suppose that $\Lambda$ is a primitive $k$-graph. For every $x \in \Lambda^{\Delta}, V^{a}(x)=\left\{y \in \Lambda^{\Delta}: x \sim_{a} y\right\}$ is dense in $\Lambda^{\Delta}$.

Proof. We need to show that, for any base element $Z(\lambda, n)$, there is a $y \in V^{a}(x) \cap Z(\lambda, n)$. Since $\Lambda$ is primitive, there is an $N \in \mathbb{N}^{k}$ such that, for every $p \geq N$ and every $u, v \in \Lambda^{0}$, there is a $v \in \Lambda^{p}$ with $s(v)=v$ and $r(v)=u$.

First, we select $m \in \mathbb{N}^{k}$ satisfying $N \leq m-n-d(\lambda)$ and $N \leq m+n$. Then, the primitive condition implies that there are $\alpha \in \Lambda^{m+n}$ and $\beta \in \Lambda^{m-n-d(\lambda)}$ such that

$$
\begin{gathered}
r(\alpha)=x(-m,-m), \quad s(\alpha)=r(\lambda), \\
s(\lambda)=r(\beta), \quad s(\beta)=x(m, m) .
\end{gathered}
$$

So, by the factorization property (cf. [8, Remarks 2.2]), there is a unique $y \in \Lambda^{\Delta}$ such that, for every $l \geq m$,

$$
\begin{gathered}
y(-l,-m)=x(-l,-m), \quad y(-m, m)=\alpha \lambda \beta, \\
y(m, l)=x(m, l) .
\end{gathered}
$$

It is trivial that $y \in Z(\lambda, n)$ from the construction, and $y \in$ $V^{a}(x)$ by Remark 4 . Therefore, the asymptotic equivalence class of any element in $\Lambda^{\Delta}$ is dense in $\Lambda^{\Delta}$.

Recall that a topological groupoid $G$ with an open range map is called minimal if the only open invariant subsets of $G^{0}$ are the empty set $\emptyset$ and $G^{0}$ itself. A subset $E$ of $G^{0}$ is called invariant if $r \circ s^{-1}(E)=E$. We refer to [9] for details.

Proposition 7. Suppose that $\Lambda$ is a primitive k-graph. Then, $G_{a}$ and $G_{a} \rtimes \mathbb{Z}^{k}$ are minimal groupoids.

Proof. We recall that the unit spaces of $G_{a}$ and $G_{a} \rtimes \mathbb{Z}^{k}$ are identified with $\Lambda^{\Delta}$ via the maps $x \mapsto(x, x)$ and $x \mapsto(x, 0, x)$, respectively. When $E$ is an invariant subset of $G_{a}^{0}$, we observe

$$
\begin{aligned}
r \circ s^{-1}(E) & =\left\{y \in \Lambda^{\Delta}: y \sim_{a} x \text { for some } x \in E\right\} \\
& =\bigcup_{x \in E} V^{a}(x)=E .
\end{aligned}
$$

We assume that there is a nontrivial open invariant subset $E$ of $G_{a}^{0}$. Then, for $z \in \Lambda^{\Delta} \backslash E$, we have $V^{a}(z) \cap E=\emptyset$ because the collection of asymptotic equivalence classes is a partition of $\Lambda^{\Delta}$. But this is a contradiction to Lemma 6 as $E$ is a nontrivial open set. So the only open invariant subsets of $G_{a}^{0}$ are the empty set and $G_{a}^{0}$, and $G_{a}$ is a minimal groupoid.

For $G_{a} \rtimes \mathbb{Z}^{k}$ and an open invariant subset $H$ of its unit space, we note that

$$
r \circ s^{-1}(H)=\bigcup_{x \in H, n \in \mathbb{Z}^{k}} V^{a}\left(\sigma^{n}(x)\right)=\bigcup_{x \in H} V^{a}(x)=H .
$$

Then, the result follows as in the case of $G_{a}$.

For an irreducible Smale space $(X, \varphi)$, Putnam and Spielberg [3] showed that groupoids defined by stable and unstable equivalence relations are essentially principal, and Hou and Chen [7] showed that $G_{a} \rtimes \mathbb{Z}$ is essentially principal. Recall that a Smale space is said to be irreducible if it is nonwandering and has a dense orbit. The basic ideas behind their proofs are the facts that $V^{a}(x)$ is at most countable for every $x$ and the set of periodic points of period $\ell$ is finite for every nonzero $\ell \in \mathbb{Z}$ (see [3, Section 5], and $[4,7.16]$ for details). We will follow [7, Section 2], and [3, Section 5], to show that $G_{a} \rtimes \mathbb{Z}^{k}$ is an essential principal.

Because of the factorization property and that $\Lambda^{m}$ is a countable set for every $m \in \mathbb{N}^{k}$, it is not difficult to check that $V^{a}(x)$ is at most countable in $k$-graphs.

Lemma 8. Let $\Lambda$ be a k-graph and $x \in \Lambda^{\Delta}$. Then, $V^{a}(x)$ is a countable set. 
Proof. Let $y \in \Lambda^{\Delta}$ be asymptotically equivalent to $x$. Then, there is an $m \in \mathbb{N}^{k}$ such that $x(m, n)=y(m, n)$ and $x(-n,-m)=y(-n,-m)$ for every $n \geq m$ by Remark 4 , and the factorization property implies that the set of finite paths

$$
\{x(-n,-m) y(-m, m) x(m, n): n \geq m\}
$$

determines a unique element in $\Lambda^{\Delta}$. It is easy to check that the uniquely determined element is $y$ as $x(-n,-m) y(-m, m) x(m, n)=y(-n, n)$ by the factorization property. So the map defined by $y \mapsto y(-m, m)$ is an injection from $V^{a}(x)$ to $\cup_{m \in \mathbb{N}^{k}} \Lambda^{2 m}$. Since $\Lambda^{m}$ is a countable set, $\cup_{m \in \mathbb{N}^{k}} \Lambda^{2 m}$ is a countable set. Hence, $V^{a}(x)$ is countable.

In irreducible Smale spaces, the set of periodic points of period $\ell \in \mathbb{Z}$, say $\operatorname{Per}_{\ell}$, is finite for every nonzero integer $\ell$ as covered in [7, Lemma 2.1] and [3, Lemma 5.2]. But Per $_{l}$ in $\left(\Lambda^{\Delta}, \sigma\right)$ may not be a finite set for some nonzero $l \in \mathbb{Z}^{k}$. For example, the 2-graph discussed in [8, Definition 1.9 and Example 6.1] has only one vertex and is irreducible, but every path has period $(1,-1) \in \mathbb{Z}^{2}$. So we need to add some extra conditions on our $k$-graphs to obtain that $\mathrm{Per}_{l}$ is finite for every nonzero $l \in \mathbb{Z}^{k}$.

Definition 9 (see [8]). Let $\Lambda$ be a $k$-graph with its twosided path space $\Lambda^{\Delta}$. A path $x \in \Lambda^{\Delta}$ is called aperiodic if $\sigma^{l} x(m, n) \neq x(m, n)$ for every $l \in \mathbb{Z}^{k} \backslash\{0\}$ and every $(m, n) \in \Delta$. We say that $\Lambda$ satisfies the aperiodic condition if, for every $v \in \Lambda^{0}$, there is an aperiodic path $x \in \Lambda^{\Delta}$ such that $x(0,0)=v$.

Observe that $\Lambda$ satisfies the aperiodic condition if and only if the set of aperiodic paths is dense in $\Lambda^{\Delta}[8$, Proposition 4.5]. So, for every $l \in \mathbb{Z}^{k}$, the $\mathbb{Z}$-action on $\Lambda^{\Delta}$ given by $j \mapsto \sigma^{j l}$ is not the identity map.

The following sublemma is definitely well known to experts, but we could not find any reference.

Sublemma 10. Suppose that $\Lambda$ is a $k$-graph with a finite $\Lambda^{0}$. If $\Lambda$ satisfies the aperiodic condition, then every isolated point in $\Lambda^{\Delta}$ is aperiodic, and the derived set of $\Lambda^{\Delta}$ is invariant under $\sigma^{l}$ for every $l \in \mathbb{Z}^{k}$.

Proof. Let $x \in \Lambda^{\Delta}$ be an isolated point. Then, there is an aperiodic point $y$ such that $x(0,0)=y(0,0)$, that is, $y \in$ $Z(x(0,0), 0)$. So we have $x=y$ because $x$ is an isolated point.

Let $z \in \Lambda^{\Delta}$ be a limit point of $\Lambda^{\Delta}$ and $\left\{z_{j}\right\}$ a sequence in $\Lambda^{\Delta}$ such that $z=\lim z_{j}$. Then, continuity of $\sigma^{l}$ implies that $\sigma^{l}(z)=\lim \sigma^{l}\left(z_{j}\right)$ so that $\sigma^{l}(z)$ is also a limit point of $\Lambda^{\Delta}$.

Since our interest is periodic points which are included in the derived set of $\Lambda^{\Delta}$ and the fact that the derived set is also compact, when there are isolated points in $\Lambda^{\Delta}$, we can consider $\mathbb{Z}^{k}$-action of $\sigma$ on the derived set instead of on $\Lambda^{\Delta}$. Thus, without loss of generality, we can assume that $\Lambda^{\Delta}$ has no isolated point.

We recall that $\sigma: \Lambda^{\Delta} \rightarrow \Lambda^{\Delta}$ is expansive with the expansive constant $\epsilon>0$ (Theorem 3 ).
Lemma 11. Suppose that $\Lambda$ is a k-graph with a finite $\Lambda^{0}$. If $\Lambda$ satisfies the aperiodic condition, then the set of periodic points of period $l$ is finite for every $l \in \mathbb{Z}^{k} \backslash\{0\}$.

Proof. For every nonzero $l \in \mathbb{Z}^{k}$, we consider an induced $\mathbb{Z}$ action $\phi$ on $\Lambda^{\Delta}$ given by $j \mapsto \phi^{j}=\sigma^{j l}$. Then, $\phi$ is also an expansive homeomorphism on $\Lambda^{\Delta}$ with the same expansive constant as that of $\sigma$, and $\operatorname{Per}_{l}=\left\{x \in \Lambda^{\Delta}: \sigma^{l}(x)=x\right\}$ is equal to $\operatorname{Fix}(\phi)=\left\{x \in \Lambda^{\Delta}: \phi(x)=x\right\}$.

Let $x$ and $y$ be two different points in $\operatorname{Fix}(\phi)$. Then, we have $\rho(x, y) \geq \epsilon$ because $\rho(x, y)=\rho\left(\phi^{j}(x), \phi^{j}(y)\right)<\epsilon$ for every $j \in \mathbb{Z}$ implies that $x=y$ by expansiveness. So the distance between any two fixed points of $\phi$ is at least $\epsilon$, and $\operatorname{Fix}(\phi)$ is a finite set as $\Lambda^{\Delta}$ is a compact set without isolated points.

Lemma 12. Let $\Lambda$ be a k-graph with a finite $\Lambda^{0}$. If $x \in \Lambda^{\Delta}$ is such that $\sigma^{l}(x) \in V^{a}(x)$ for some $l \in \mathbb{Z}^{k} \backslash\{0\}$, then

(1) the sequence $\left\{\sigma^{j l}(x): j \in \mathbb{N}\right\}$ converges to an element, say $x_{1}$, in $\operatorname{Per}_{l}$ as $j \rightarrow \infty$ and $x \in V^{s}\left(x_{1}\right)$,

(2) the sequence $\left\{\sigma^{-j l}(x): j \in \mathbb{N}\right\}$ converges to an element, say $x_{2}$, in $\operatorname{Per}_{l}$ as $j \rightarrow \infty$ and $x \in V^{u}\left(x_{2}\right)$.

Proof. Convergence of $\left\{\sigma^{j l}(x): j \in \mathbb{N}\right\}$ to $x_{1} \in \operatorname{Per}_{l}$ is equivalent to $\left(\Lambda^{\Delta}, \sigma\right)$ being an irreducible Smale space, so we refer to $[3,7]$ for details.

To show that $x \in V^{s}\left(x_{1}\right)$, we need to find an $m \in \mathbb{Z}^{k}$ such that $x(m, n)=x_{1}(m, n)$ for every $n \geq m$. Let $m \in \mathbb{N}^{k}$ be the number given by $\sigma^{l}(x) \sim{ }_{a} x$ so that $x(m, n)=\sigma^{l}(x)(m, n)$ and $x(-n,-m)=\sigma^{l}(-n,-m)$ for every $n \geq m$.

Because $\sigma^{j l}(x)$ converges to $x_{1}$ as $j \rightarrow \infty$, for every open neighborhood $U$ of $x_{1}$, there is an $N \in \mathbb{N}$ such that $j \geq N$ implies $\sigma^{j l}(x) \in U$. For

$$
U=Z\left(x_{1}(m, n), m\right)=\left\{y \in \Lambda^{\Delta}: y(m, n)=x_{1}(m, n)\right\},
$$

let $N \in \mathbb{N}$ be such that $\sigma^{N l}(x) \in Z\left(x_{1}(m, n), m\right)$, that is,

$$
\sigma^{N l}(x)(m, n)=x(m+N l, n+N l)=x_{1}(m, n) .
$$

Since $x_{1}$ is a periodic point of period $l$, we have $\sigma^{-N l}\left(x_{1}\right)(m, n)=x_{1}(m, n)=x(m, n)$ for every $n \geq m$. Therefore, $x$ is stably equivalent to $x_{1}$.

The case of $x \in V^{u}\left(x_{2}\right)$ is analogous.

Lemma 13. Suppose that $\Lambda$ is a k-graph and that $\Lambda^{0}$ is a finite set. If $\Lambda$ satisfies the aperiodic condition, then the set

$$
\left\{x \in \Lambda^{\Delta}: \sigma^{l}(x) \in V^{a}(x) \text { for some } l \in \mathbb{Z}^{k} \backslash\{0\}\right\},
$$

is a countable set.

Proof. Let $x \in \Lambda^{\Delta}$ be such that $\sigma^{l}(x) \in V^{a}(x)$ for some $l \epsilon$ $\mathbb{Z}^{k} \backslash\{0\}$. First we notice that, by Lemma 12, we have $x \sim{ }_{s} x_{1}$ and $x \sim{ }_{u} x_{2}$ so that $V^{s}(x)=V^{s}\left(x_{1}\right)$ and $V^{u}(x)=V^{u}\left(x_{2}\right)$. Hence, $V^{s}\left(x_{1}\right) \cap V^{u}\left(x_{2}\right)=V^{a}(x)$ is a countable set by Lemma 8 . 
Lemma 12 implies that $x \in V^{s}\left(x_{1}\right) \cap V^{u}\left(x_{2}\right)$ so that the set

$$
\left\{x \in \Lambda^{\Delta}: \sigma^{l}(x) \in V^{a}(x) \text { for some } l \in \mathbb{Z}^{k} \backslash\{0\}\right\},
$$

is a subset of

$$
\bigcup_{l \in \mathbb{Z}^{k}} \bigcup_{x_{1}, x_{2} \in \operatorname{Per}_{l}} V^{s}\left(x_{1}\right) \cap V^{u}\left(x_{2}\right) .
$$

The union is a countable union by Lemma 11 , and $V^{s}\left(x_{1}\right) \cap$ $V^{u}\left(x_{2}\right)$ is a countable set by the previous paragraph and Lemma 8. This completes the proof.

Recall that a topological groupoid $G$ with an open range map is called essentially principal if $G$ is locally compact and, for every closed invariant subset $F$ of its unit space $G^{0},\{u \in$ $\left.F: r^{-1}(u) \cap s^{-1}(u)=\{u\}\right\}$ is dense in $F$ (see [9] for details).

Theorem 14. Suppose that $\Lambda$ is a $k$-graph and that $\Lambda^{0}$ is a finite set. Then, $\Lambda$ satisfies the aperiodic condition if and only if $G_{a} \rtimes$ $\mathbb{Z}^{k}$ is an essentially principal groupoid.

Proof. Let $B=\left\{u \in\left(G_{a} \rtimes \mathbb{Z}^{k}\right)^{0}: s^{-1}(u) \cap r^{-1}(u)=\{u\}\right\}$, collection of points of $\left(G_{a} \rtimes \mathbb{Z}^{k}\right)^{0}$ whose isotropy group is trivial. We observe that, for $u=(x, 0, x) \in\left(G_{a} \rtimes \mathbb{Z}^{k}\right)^{0}, u \notin B$ if and only if there is an $l \in \mathbb{Z}^{k} \backslash\{0\}$ such that $\sigma^{l}(x) \sim{ }_{a} x$. And every element in $B$ is aperiodic because a periodic point $y \in$ $\Lambda^{\Delta}$ with period $l$ satisfies $\sigma^{l}(x)=x$, that is, $\sigma^{l}(x) \in V^{a}(x)$ and $x \notin B$.

If $\Lambda$ satisfies the aperiodic condition, then $B^{c}$ is a countable set by Lemma 13 so that $B$ is a dense subset of $\left(G_{a} \rtimes \mathbb{Z}^{k}\right)^{0}$. Therefore $G_{a} \rtimes \mathbb{Z}^{k}$ is an essentially principal groupoid.

If $G_{a} \rtimes \mathbb{Z}^{k}$ is essentially principal, then $B$ is dense in $\Lambda^{\Delta}$, and the set of aperiodic paths is dense in $\Lambda^{\Delta}$ by the previous paragraph. Hence, for every $v \in \Lambda^{0}$, the base element $Z(v, 0)$ must contain aperiodic paths. Thus, $\Lambda$ satisfies the aperiodic condition.

Before we go to the main theorem, let us check that our groupoids $G_{a}$ and $G_{a} \rtimes \mathbb{Z}^{k}$ are amenable so that we do not need to use reduced groupoid algebras: let $G_{s}$ and $G_{u}$ be the locally compact principal groupoids defined by the stable and unstable equivalence relations, respectively, on $\Lambda^{\Delta}$. Kumjian and Pask showed that $G_{s}$ and $G_{u}$ are amenable in [1, Theorem 5.3]. Then, proof of the following lemma is exactly the same as [7, Lemma 2.5] and [1, Remark 5.8].

Lemma 15. Suppose that $\Lambda$ is a k-graph. Then, $G_{a}$ and $G_{a} \rtimes \mathbb{Z}^{k}$ are amenable in the sense of Renault.

Now we apply [9, Proposition 4.6], Proposition 7, and Theorem 14 to obtain simplicity of $C^{*}$-algebras of $G_{a}$ and $G_{a} \rtimes \mathbb{Z}^{k}$. Remark that a primitive $k$-graph with our standing assumption automatically has a finite $\Lambda^{0}$.

Theorem 16. Suppose that $\Lambda$ is a primitive $k$-graph. Then, the asymptotic $C^{*}$-algebras $A(\Lambda)$ generated by $G_{a}$ is a simple nuclear $C^{*}$-algebra. If in addition $\Lambda$ satisfies the aperiodic condition, then the asymptotic Ruelle algebra $R_{a}(\Lambda)$ generated by $G_{a} \rtimes \mathbb{Z}^{k}$ is also a simple nuclear $C^{*}$-algebra.

\section{Acknowledgment}

The author would like to express his deep gratitude to referees for their kind suggestions.

\section{References}

[1] A. Kumjian and D. Pask, "Actions of $\mathbb{Z}^{k}$ associated to higher rank graphs," Ergodic Theory and Dynamical Systems, vol. 23, no. 4, pp. 1153-1172, 2003.

[2] I. F. Putnam, " $C^{*}$-algebras from Smale spaces," Canadian Journal of Mathematics, vol. 48, no. 1, pp. 175-195, 1996.

[3] I. F. Putnam and J. Spielberg, "The structure of $C^{*}$-algebras associated with hyperbolic dynamical systems," Journal of Functional Analysis, vol. 163, no. 2, pp. 279-299, 1999.

[4] D. Ruelle, Thermodynamic Formalism, vol. 5 of Encyclopedia of Mathematics and Its Applications, Addison-Wesley, 1978.

[5] D. Ruelle, "Noncommutative algebras for hyperbolic diffeomorphisms," Inventiones Mathematicae, vol. 93, no. 1, pp. 1-13, 1988.

[6] J. Cuntz and W. Krieger, "A class of $C^{*}$-algebras and topological Markov chains," Inventiones Mathematicae, vol. 56, no. 3, pp. 251-268, 1980.

[7] C. Hou and X. Chen, "A note on the ideals of groupoid $C^{*}$-algebras from Smale spaces," Bulletin of the Australian Mathematical Society, vol. 64, no. 2, pp. 271-279, 2001.

[8] A. Kumjian and D. Pask, "Higher rank graph $C^{*}$-algebras," New York Journal of Mathematics, vol. 6, pp. 1-20, 2000.

[9] J. Renault, A Groupoid Approach to $C^{*}$-Algebras, vol. 793 of Lecture Notes in Mathematics, Springer, Berlin, Germany, 1980. 


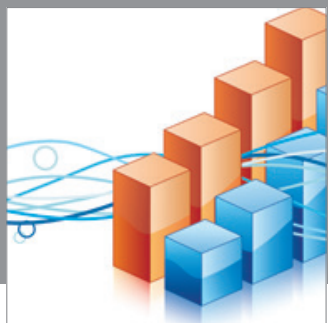

Advances in

Operations Research

mansans

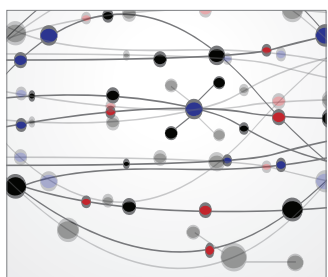

The Scientific World Journal
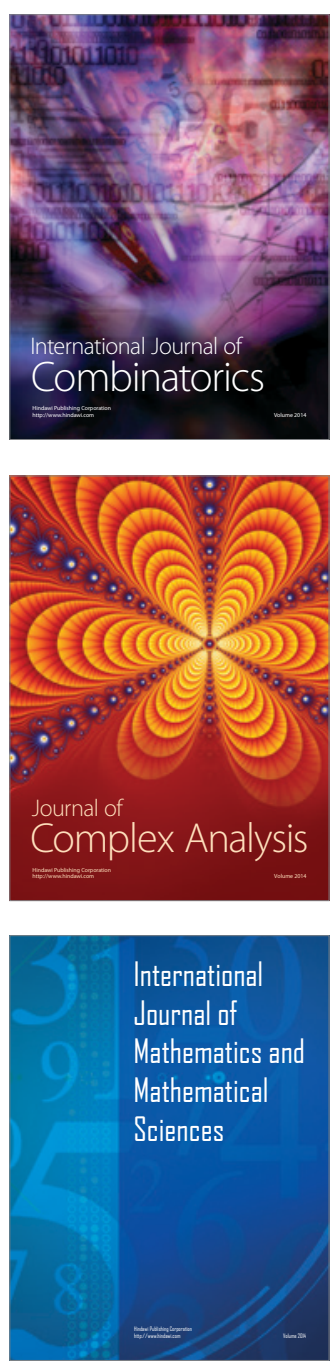
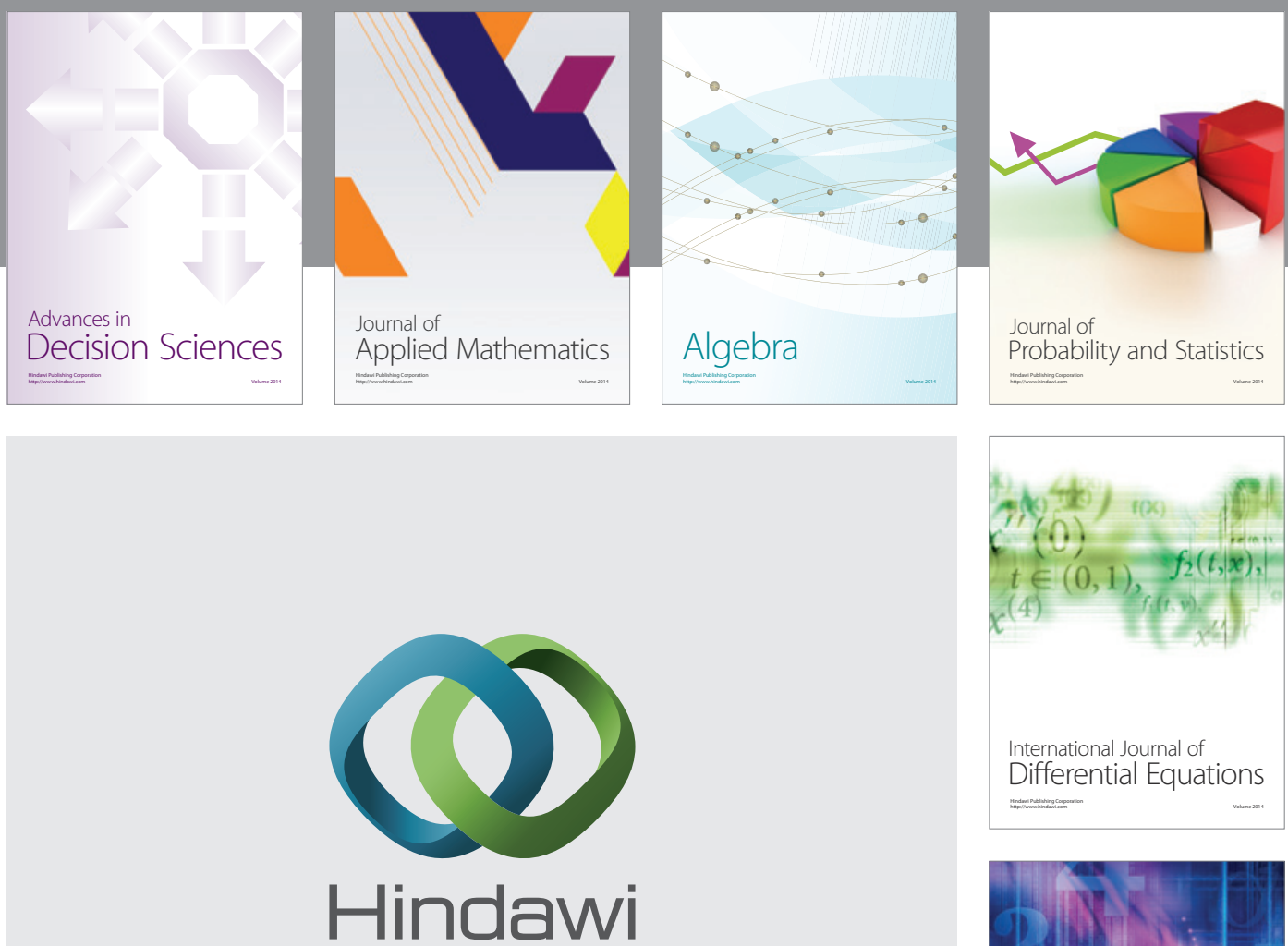

Submit your manuscripts at http://www.hindawi.com
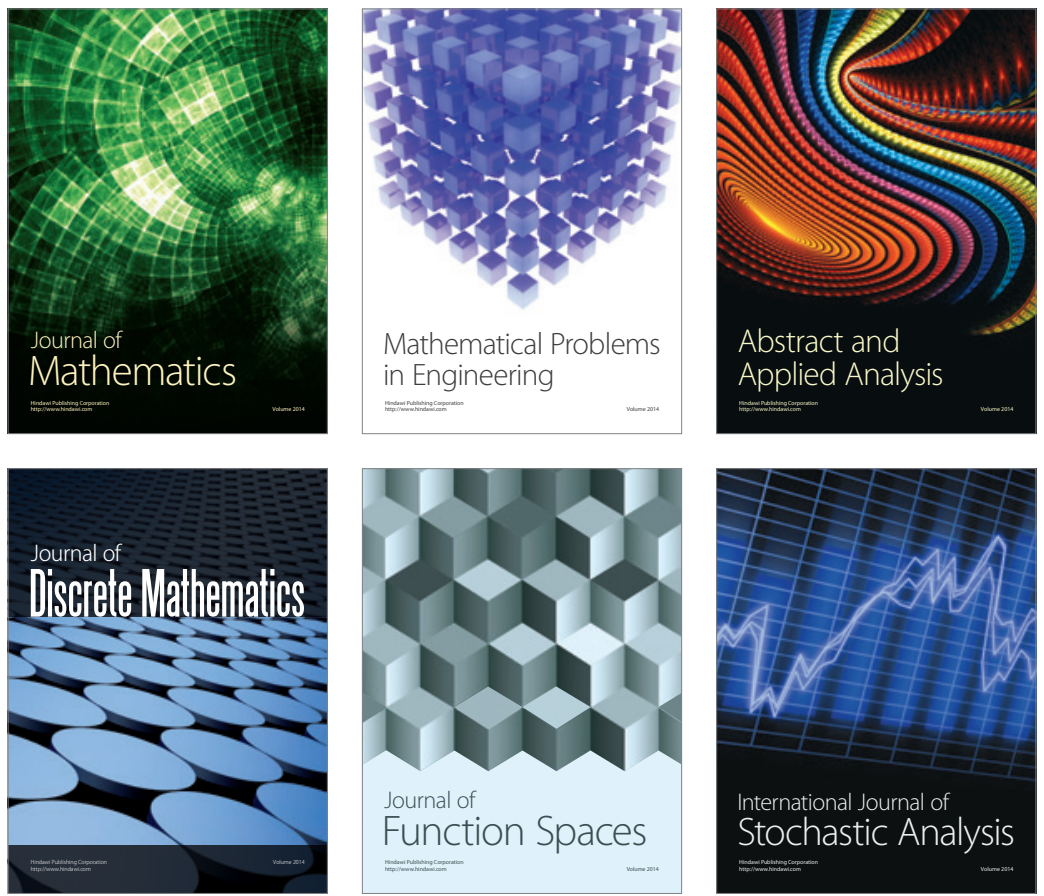

Journal of

Function Spaces

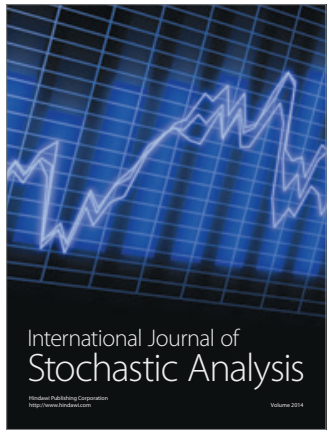

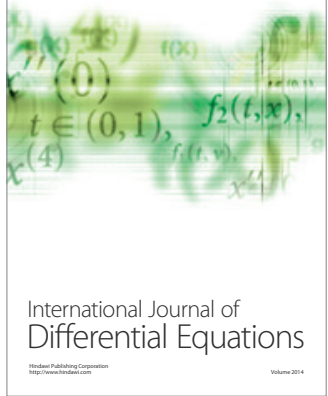
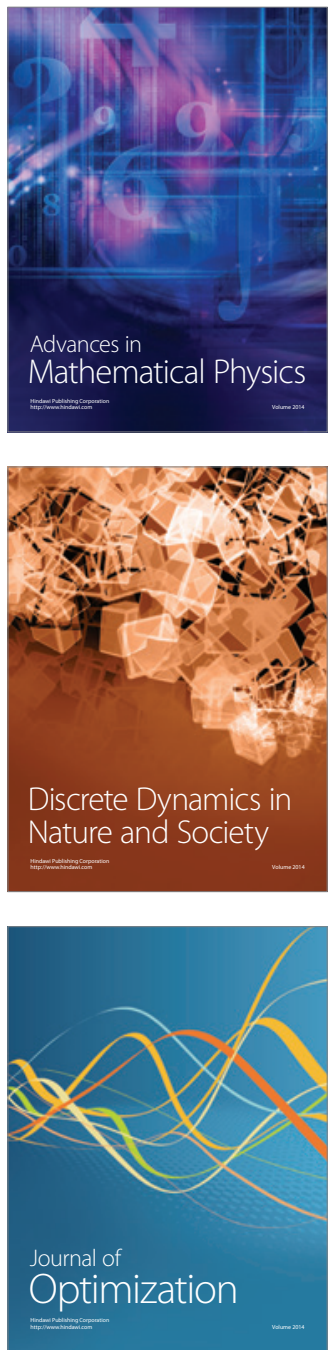